\title{
Examining the neural antecedents of tics in Tourette syndrome using electroencephalography
}

\author{
Barbara Morera Maiquez', Georgina M. Jackson ${ }^{2}$ and Stephen R. \\ Jackson*1,2 \\ 'School of Psychology, University of Nottingham, UK \\ ${ }^{2}$ Institute of Mental Health, School of Medicine, University of Nottingham, UK
}

\begin{abstract}
Tourette syndrome (TS) is a neurodevelopmental disorder characterized by the occurrence of motor and vocal tics. TS is associated with cortical-striatal-thalamiccortical circuit dysfunction and hyper-excitability of cortical limbic and motor regions that lead to the occurrence of tics. Importantly, individuals with TS often report that their tics are preceded by premonitory sensory/urge phenomena (PU) that are described as uncomfortable bodily sensations that precede the execution of a tic and are experienced as an urge for motor discharge. While tics are most often referred to as involuntary movements, it has been argued by some that tics should be viewed as voluntary movements that are executed in response to the presence of PU. To investigate this issue further, we conducted a study using electroencephalography (EEG). We recorded movement-related EEG (mu- and beta-band oscillations) during ( $\mathrm{I}$ ) the immediate period leading up to the execution of voluntary movements by a group of individuals with TS and a group of matched healthy control participants, and (2) the immediate period leading up to the execution of a tic in a group of individuals with TS. We demonstrate that movementrelated mu and beta oscillations are not reliably observed prior to tics in individuals with TS. We interpret this effect as reflecting the greater involvement of a network of brain areas, including the insular and cingulate cortices, the basal ganglia and the cerebellum, in the generation of tics in TS. We also show that beta-band desynchronization does occur when individuals with TS initiate voluntary movements, but, in contrast to healthy controls, desynchronization of mu-band oscillations is not observed during the execution of voluntary movements for individuals with TS. We interpret this finding as reflecting a dysfunction of physiological inhibition in TS, thereby contributing to an impaired ability to suppress neuronal populations that may compete with movement preparation processes.
\end{abstract}

Tourette syndrome (TS) is a neurological disorder of childhood onset that is characterized by the presence of chronic vocal and motor tics (Cohen, Leckman, \& Bloch, 2013). Tics are repetitive, stereotyped behaviours that occur with a limited duration (Cohen et al., 2013). Motor tics can be simple or complex in appearance, ranging from repetitive movements to coordinated action sequences. Verbal tics can consist of repetitive sounds, words or utterances, the production of inappropriate or obscene utterances, or the

This is an open access article under the terms of the Creative Commons Attribution License, which permits use, distribution and reproduction in any medium, provided the original work is properly cited.

*Correspondence should be addressed to Stephen R. Jackson, School of Psychology, The University of Nottingham, Nottingham, NG7 2RD, UK (email: Stephen.jackson@nottingham.ac.uk). 
repetition of another's words. Tics occur in bouts, typically many times in a single day, and are the most common form of movement disorder in children.

The volitional nature of tics has been a topic of debate (Cavanna et al., 2017). While many refer to tics as involuntary movements (e.g., Ganos et al., 2015), others have argued that tics are instead voluntary and occur in response to so-called 'premonitory urges' (Leckman, Bloch, Scahill, \& King, 2006). Thus, the majority of individuals with TS report that their tics are often preceded by premonitory sensory/urge phenomena (PU) that are described as uncomfortable cognitive or bodily sensations that occur prior to the execution of a tic and are experienced as a strong urge for motor discharge (Cohen et al., 2013). Individuals who experience PU often report that: these experiences are more bothersome than their tics; that expressing their tics give them relief from, and temporarily abolishes, their PU; and that they would not exhibit tics if they did not experience PU. For this reason, it has been proposed that PU should be considered as the driving force behind the occurrence of tics, and that tics are a learnt response to the experience of PU (Cavanna et al., 2017; Leckman et al., 2006).

One important argument for distinguishing tics from volitional movements is the finding that motor tics do not exhibit the peri-movement electrical (EEG) potentials that are observed immediately before volitional movements (Obeso et al., 1981). Specifically, voluntary movements are typically associated with distinct pre-movement 'readiness' (RP), motor (MP), and post-movement potentials. The pre-movement (RP) potential is represented by a negative wave beginning about 1.5 or $2 \mathrm{~s}$ before the movement which leads to a rapid rise in negativity at around $500 \mathrm{~ms}$ before movement onset. The MP is represented by a positive peak and occurs with movement onset and is localized to the contralateral primary sensorimotor cortex (SMC). Previous studies have shown that while voluntary movements produced by individuals with TS are preceded by the normal perimovement electrical potentials as observed in typically developing individuals (Karp, Porter, Toro, \& Hallett, 1996; Obeso et al., 1981; however, see Zapparoli, Macerollo, Joyce, Martino, \& Kilner, 2019) these potentials are reported to be absent prior to, and at the time of, motor tics (Obeso et al., 1981). Note, however, that subsequent studies have reported a more nuanced picture, indicating that tic generation was associated with an RP in only a minority of patients (Karp et al., 1996; van der Salm, Tijssen, Koelman, \& van Rootselaar, 2012), or in a small number of individual patients (Duggal \& Nizamie, 2002).

Volitional movements lead to robust modulation of movement-related brain oscillations in the mu $(8-12 \mathrm{~Hz})$ and beta $(13-30 \mathrm{~Hz})$ frequency bands. Specifically, there is a substantial decrease in power (event-related desynchronization - ERD) in both frequency bands that typically commences shortly before movement onset, and is sustained for the period of movement execution. After the movement is terminated, there is a substantial increase in power (event-related synchronization - ERS) for both rhythms. The ERD is thought to reflect cortical activation, while ERS is thought to reflect reduced cortical excitability or inhibition. This is supported by TMS studies, which have shown that there is an increase in motor excitability just prior to a movement (80-100 ms before movement) (Chen, Yaseen, Cohen, \& Hallett, 1998), during a movement (Chen et al., 1998), and that motor cortex excitability then decreases $\sim 500-1,000 \mathrm{~ms}$ after the movement has completed (Chen, Corwell, \& Hallett, 1999). For beta-band oscillations, this is often referred to as the post-movement beta rebound (PMBR). It has been suggested that these movement-related brain oscillations, particularly the beta-band oscillations, reflect an internal estimate of the likelihood of the need for a novel voluntary action, and in effect signal motor readiness (Jenkinson \& Brown, 2011). If this is the case then one might make the following predictions. If tics are indeed voluntary movements executed intentionally 
to relieve PU, then we would expect to see clear evidence for movement-related ERD when individuals with TS execute volitional movements, and also when they tic. By contrast, if tics are essentially involuntary movements, then we would expect to see clear evidence for movement-related ERD when individuals with TS execute volitional movements, but not necessarily when they execute motor tics. The current study investigated this issue using EEG-recording techniques.

\section{Methods: Study I. Neural antecedents of volitional movement \\ Participants}

Seventeen individuals (seven females; aged between 12 and 51 years; mean age of 23 years) with a confirmed clinical diagnosis of TS were recruited to this study. Three participants were left-handed. All of the participants recruited to this study reported that they typically experienced premonitory urge (PU) phenomena prior to their tics. This is confirmed by their PUTS scores reported in Table 1. Participants with co-occurring neuropsychiatric conditions were not excluded. Data from two TS participants were excluded due to excessive noise caused by the frequent and intense tics during EEG recording. The remaining 15 participants included seven females aged between 12 and 35 years (mean age $=20.3$ years). Three participants were left-handed. Details from TS participants can be found in Table 1. Fifteen individuals (nine females; aged between 19 and 28 years; mean age of 22.5 years) were recruited as controls. One participant was lefthanded. All participants gave informed consent, and the study was approved by an appropriate local ethical review committee.

\section{Devices}

The visual stimuli were presented on a computer screen (Samsung S23A700D, screen resolution: $1,920 \times 1,080)$. Participants made responses on an Apple Pro Keyboard M7803 connected to a Mac Pro computer running High Sierra (v 10.13.6) via USB cable. The task was controlled by PsychoPy2 (v1.90.3) script coded using python 2 on the Mac Pro.

\section{Experimental procedure}

Participants performed a manual choice Go/NoGo task using the index finger of their dominant hand. Each trial consisted of a fixation cross, presented in the centre of the computer monitor, that disappeared after $1000 \mathrm{~ms}$, which was followed by the appearance of the cue stimulus (either a square or a circle) that appeared in the centre of the computer monitor. The task consisted of pressing the response key (space bar) as quickly as possible whenever either a yellow circle or a blue square appeared in the centre of the screen (i.e., the Go condition), or withholding a response whenever a blue circle or a yellow square appeared (i.e., NoGo condition). $70 \%$ of the trials were Go trials and $30 \%$ of the trials were NoGo trials. The diameter of both stimuli was $5 \mathrm{~cm}$. When no response was made, the next trial appeared after $1 \mathrm{~s}$. When a response was made, the next trial commenced.

Participants were seated approximately $70 \mathrm{~cm}$ away from the screen. There were 400 trials and a short break was offered every 100 trials. After every break, a reminder of the instructions of the task appeared on the screen. The task did not take longer than $15 \mathrm{~min}$. TS participants were told not to suppress their tics. 


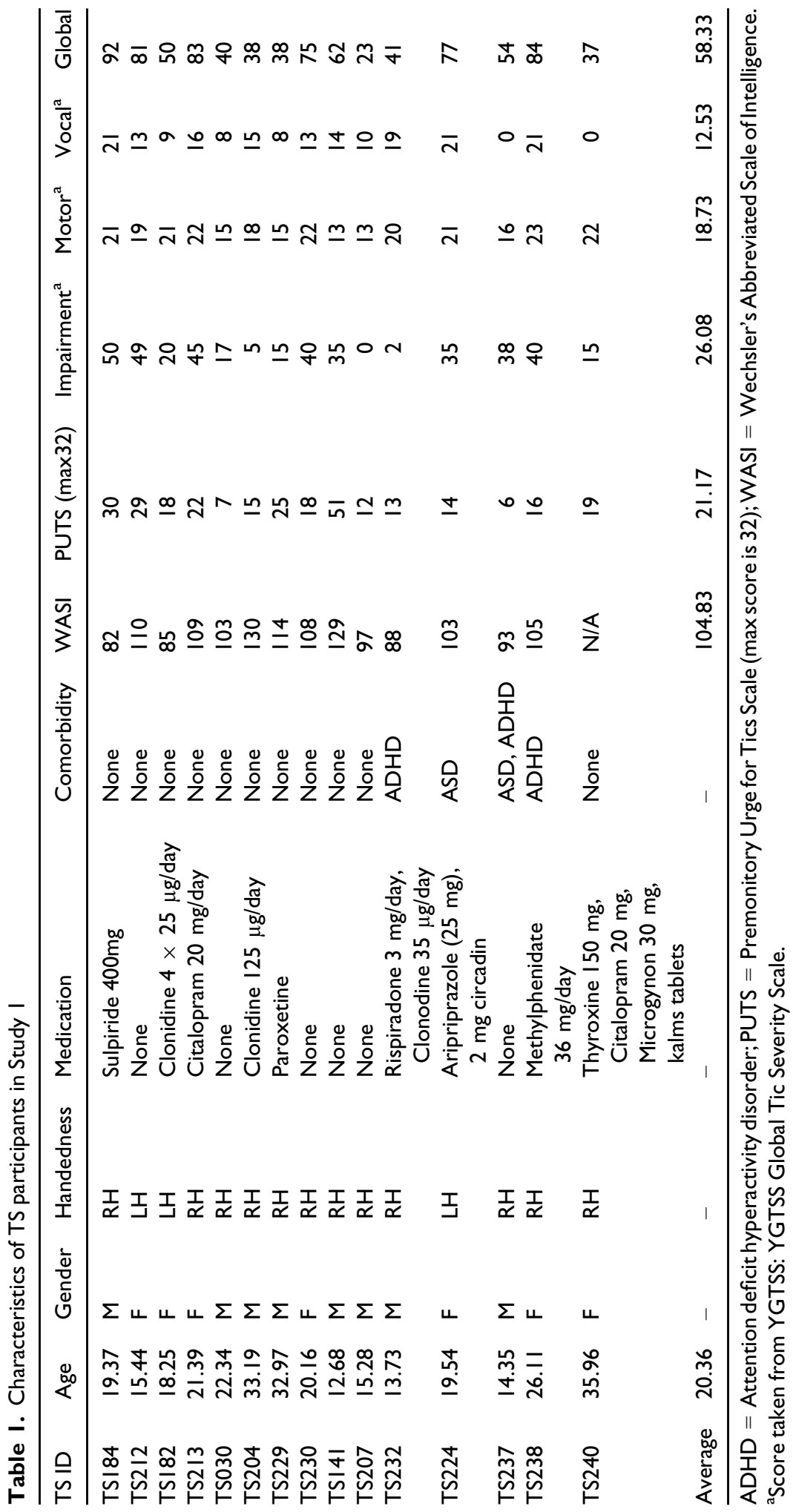


Before the study commenced, participants completed a set of 20 practice trials. If at least $90 \%$ of those trials were performed correctly, the subject proceeded to start the actual study; if less than $90 \%$ of those trials were performed correctly, the subject continued to perform another practice of 20 trials until at least $90 \%$ of those trials were performed correctly.

\section{Electroencephalography (EEG) recording}

EEG data were recorded from 64 electrodes using a BioSemi (Amsterdam, The Netherlands) Active Two System. Data were recorded with a sampling rate of $1024 \mathrm{~Hz}$ which was subsequently down-sampled to $128 \mathrm{~Hz}$. The impedance of the electrodes was kept under $30 \mu \mathrm{V}$ for all participants. Reference electrodes were placed on the left and right mastoids. Bipolar vertical and horizontal electrooculography (EOG) was also recorded.

\section{EEG data analyses}

EEGLAB 14 (14.1.1) was used to pre-process the EEG data.

Data were low-pass filtered at $45 \mathrm{~Hz}$ and high-pass filtered at $1 \mathrm{~Hz}$ using Finite Impulse Response (FIR). Channels showing aberrant behaviour were deleted, and noisy channels were interpolated using spherical interpolation. No more than four channels per subject were interpolated for the HS group, and no more than five channels per subject were

(a)

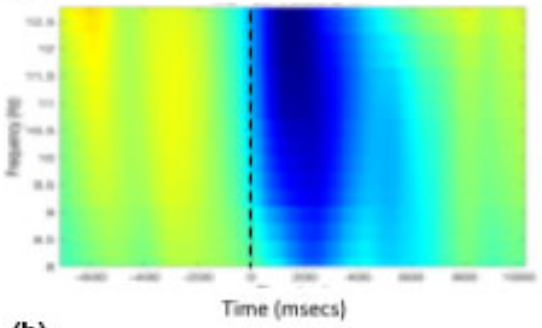

(b)

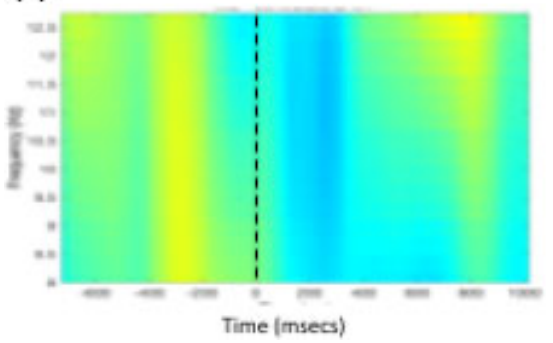

(c)

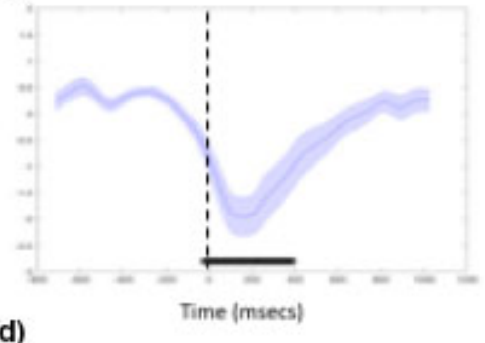

(d)

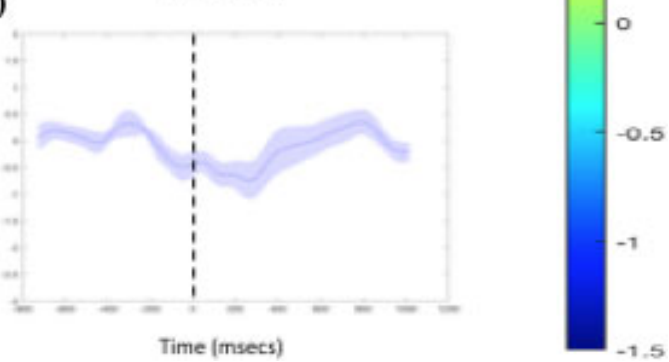

Figure I. Time-frequency plots showing mu-band frequency power recorded from the scalp over the contralateral sensorimotor area. (A) Data from healthy controls. (B) Data from individuals with TS. $12 \mathrm{~Hz}$ ERSP values recorded from the scalp over the contralateral sensorimotor area. (C) Data from healthy controls. (D) Data from individuals with TS. The vertical broken black line in each image at time zero on the $x$-axis indicates the time at which the button press occurred. The black horizontal line indicates individual time points at which the ERSP values significantly differed from zero $\left(p<.05^{\text {FDR-corrected }}\right)$. The shading in $\mathrm{C}$ and $\mathrm{D}$ represents the standard error. 
interpolated for the TS group. Automatic artefact removal (AAR) was used to remove EOG artefacts using a recursive least squares regression.

Data were standardized ( $z$-scores) to produce data with a mean of 0 and standard deviation to 1. As a result, no baselining of the data was required. Epochs showing abnormal trends or excessive noise were rejected: those epochs showing a signal amplitude at $\pm 100 \mu \mathrm{V}$ in one or more channels were rejected; those epochs with signal slopes exceeding a threshold of $50 \mu \mathrm{V}$ in one or more channels were rejected; those epochs with five times the standard deviation in the probability distribution were rejected; those epochs which their kurtosis statistic was larger than five times of the standard deviation of the data were rejected. An average of 255 epochs was obtained in the HS group, and an average of 172 epochs was obtained in the TS group.

Independent component analysis (ICA) were conducted, and artefacts were identified by visual inspection and through the use of the multiple artefact rejection algorithm (MARA).

Analyses of the EEG data recorded from scalp sensors located over the motor cortex contralateral to the responding hand (i.e., C3 for right-handed participants and C4 for lefthanded participants) yielded mean event-related potentials (ERPs) and mean event-related spectral perturbation (ERSP) for correct Go trials. All NoGo trials, and Go trials in which no response was recorded, were discarded from the analyses. In addition, and importantly, all epochs in which the participant was recorded (observed) to be ticcing were discarded from the analyses. Time-frequency analyses were performed using Fast Fourier Transform (FFT). There were a total of 256 data points. The moving window length was of $250 \mathrm{~ms}$, and the frequency resolution was $0.25 \mathrm{~Hz}$.

For each correct Go trial, a time window of -0.8 to $1.2 \mathrm{~s}$ was constructed and timelocked to each button press. In each case, zero seconds indicated the time of each button press. Statistical analyses were performed separately for mu $(8-13 \mathrm{~Hz})$ and beta (13-

(a)

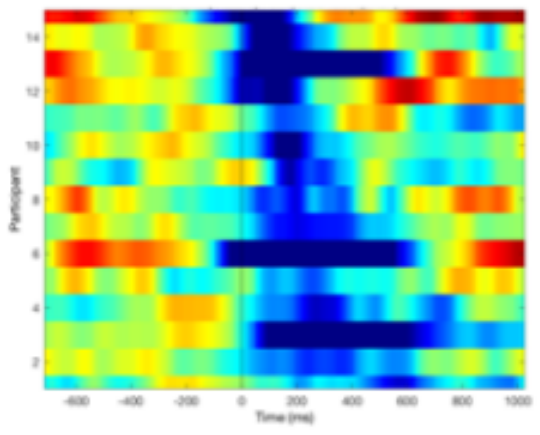

(b)

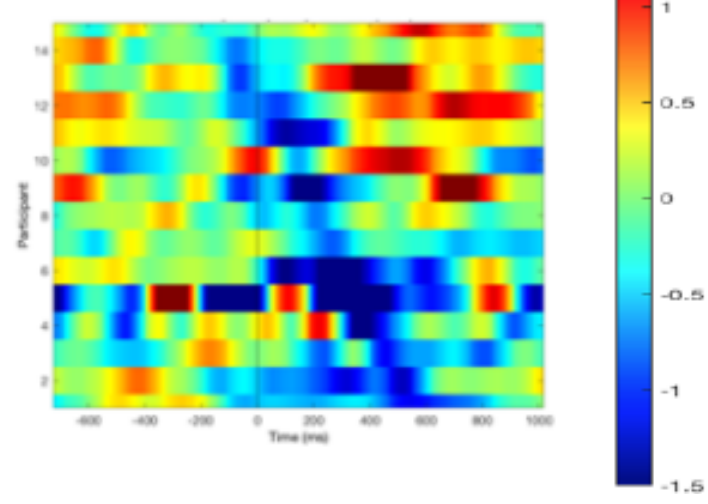

Figure 2. Individual mu-band peak event-related desynchronization (ERD) data were rank-ordered from the earliest (top) occurrence to the latest (bottom) occurrence. (A) Data from each of the healthy control group is located close to the time of movement onset. (B) Data from the TS group is much more variable. While the mean time of maximum ERD does not differ statistically across groups, the variability is much larger in the TS group (coefficient of variation: $\mathrm{HC}$ group $=0.53$; $\mathrm{TS}$ group $=0.95$ ). 
(a)
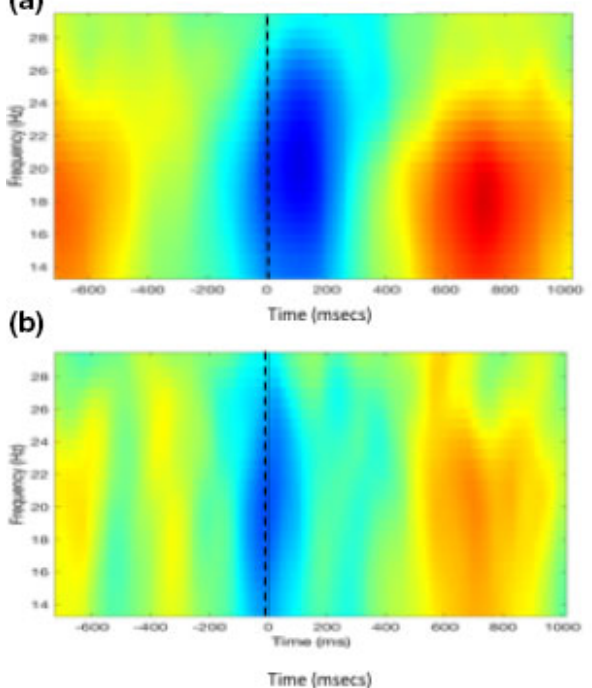

(c)

(d)

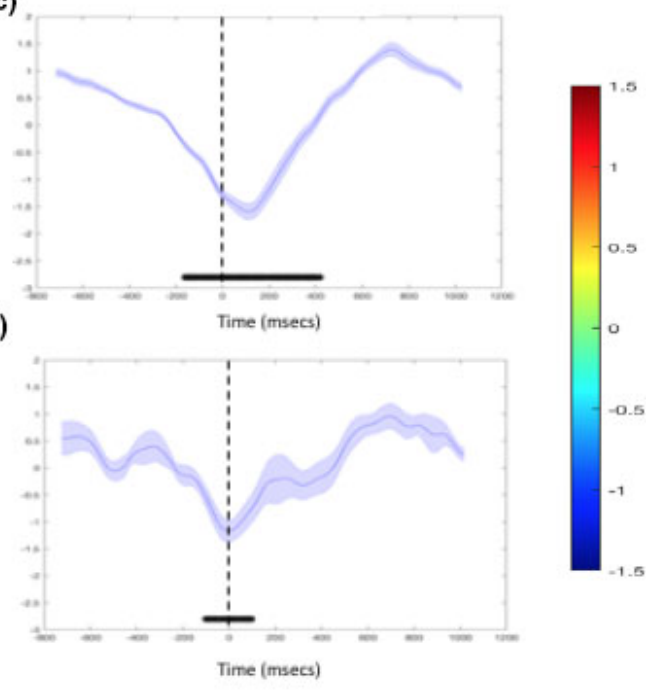

Figure 3. Time-frequency plots showing beta-band frequency power recorded from the scalp over the contralateral sensorimotor area. (A) Data from healthy controls. (B) Data from individuals with $\mathrm{TS} .19 \mathrm{~Hz}$ ERSP values recorded from the scalp over the contralateral sensorimotor area. (C) Data from healthy controls. (D) Data from individuals with TS. The vertical broken black line in each image at time zero on the $x$-axis indicates the time at which the button press occurred. The black horizontal line indicates individual time points at which the ERSP values significantly differed from zero $\left(p<.05^{\text {FDR-corrected }}\right)$. The shading in $C$ and $D$ represents the standard error.

$30 \mathrm{~Hz}$ ) frequency responses to test the null hypothesis that the sample data are from a population with mean equal to zero at the $5 \%$ significance level. One-sample t-tests, with a test variable of 0 , were conducted at each time point correcting for the multiple comparisons using false discovery rate (FDR; Benjamini and Hochberg, 1995). Twosample $t$-tests were also conducted to test for between-group differences.

\section{Results}

\section{Mu-band ERSP analyses}

Time-frequency analyses were conducted for both mu-band and beta-band frequencies. Mu-band time-frequency ERSP data for both the TS group and healthy controls are presented in Figure 1. Inspection of this figure indicates that, for the healthy control group (Figure 1A), there is a strong reduction in mu-band power (desynchronization) that coincides with the movement onset, and statistical analysis using one-sample t-tests (Figure 1C) confirm that the standardized (Z) ERSP values differ from zero $\left(p<.05^{\mathrm{FDR}-}\right.$ corrected). This difference lasts for $390 \mathrm{~ms}$. By contrast, inspection of Figure 1B suggests that mu-band desynchronization is much weaker in the TS group and occurs sometime after movement onset. This is confirmed by the statistical analyses (Figure 1D) which demonstrated that at no time did standardized mu-band power (ERSP) differ significantly from zero.

To investigate this further, we examined individual event-related desyncronization (ERD) responses as follows. For each individual, we calculated the time of the maximum 
(a)

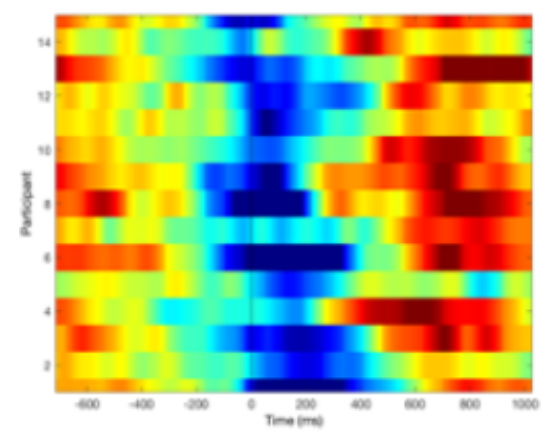

(b)

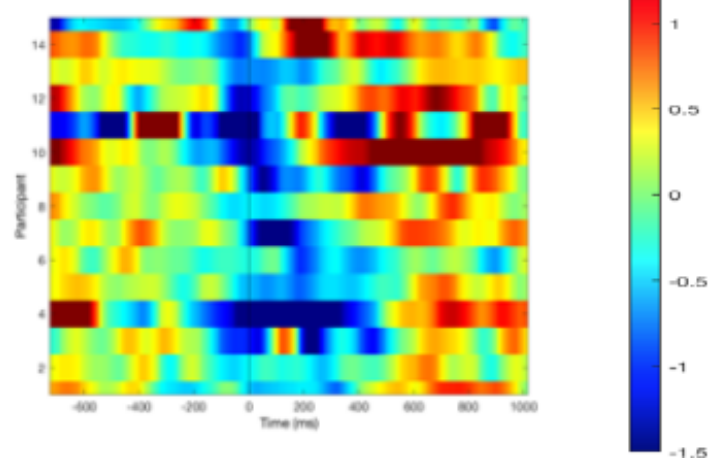

Figure 4. Individual beta-band peak event-related desynchronization (ERD) data were rank-ordered from the earliest (top) occurrence to the latest (bottom) occurrence. (A) Data from each of the healthy control group is located close to the time of movement onset. (B) Data from the TS group is much more variable. While the mean time of maximum ERD does not differ across groups, the variability is much greater in the TS group (coefficient of variation: $\mathrm{HC}$ group $=0.78$; TS group $=1.15$ ).

ERD for that individual. The resultant rank-ordered data are displayed in Figure 2. Further analyses revealed that the mean time of maximum ERD was $242 \mathrm{~ms}$ ( $\pm 129 \mathrm{~ms}$ ) after movement onset for the control group and $227 \mathrm{~ms}( \pm 215 \mathrm{~ms})$ for the TS group. An independent group t-test revealed that these means did not differ statistically $(p>.1)$. It is of interest to note that the timing of the ERD appeared to be more variable in the TS group, that is the coefficient of variation for the TS group was almost twice that of the CS group; however, this difference in variability did not meet conventional levels of statistical significance (Figure 2: coefficient of variation; HC group $=0.53$; TS group $=0.95$; $Z=1.45, p>.1)$.

\section{Beta-band ERSP analyses}

Beta-band time-frequency ERSP data for the TS and healthy control groups are presented in Figure 3. Inspection of this figure indicates that, for the healthy control group (Figure $3 \mathrm{~A}$ ), there is a strong reduction in beta-band power (ERD) that begins in advance of movement onset and is sustained for several hundred milliseconds after movement onset. Statistical analysis, using one-sample t-tests (Figure 3C), confirms that the standardized $(Z)$ ERSP values differ significantly $\left(p<.05^{\text {FDR-corrected }}\right)$ from zero throughout this period. This difference lasts for $468 \mathrm{~ms}$. Interestingly, and in contrast to the results for mu-band power, inspection of Figure 3B indicates that beta-band ERD also occurs prior to movement onset for the TS group and lasts for $179 \mathrm{~ms}$ (Figure 3D).

Again, to investigate this further, we examined individual event-related desyncronization (ERD) responses. For each individual, we calculated the time of the maximum ERD for that individual. The resultant rank-ordered data are displayed in Figure 4. Further analyses revealed that the mean time of maximum ERD was $111 \mathrm{~ms}( \pm 86 \mathrm{~ms})$ after movement onset for the control group and $110 \mathrm{~ms}( \pm 128 \mathrm{~ms})$ for the TS group. An independent group t-test revealed that these means did not differ statistically $(p>.1)$. However, it 
should be noted that the timing of the ERD again appeared to be more variable in the TS group (Figure 4: coefficient of variation; HC group $=0.78$; TS group $=1.15 ; p>.1$ ), but again this difference in variability did not meet conventional levels of statistical significance. We suggest that this issue should be explored further in a larger sample.

\section{Between-group analyses of mu and beta power}

To test directly whether mu-band and beta-band ERD were significantly different between groups, we carried out the following analyses. For each individual, and for each group, we calculated the mean ERSP value within a 1,000 ms time window centred around movement onset (i.e., $500 \mathrm{~ms}$ before movement onset to $500 \mathrm{~ms}$ after movement onset). We then tested the between-group difference between the mean ERD using an independent group t-test. Separate analyses were conducted for mu and beta power. The analysis revealed that there was no significant between-group difference in mean beta-band power (control group $=-0.31( \pm 0.43)$ decibels, TS group $=-0.16( \pm 0.51) ; t$ $(28)=-0.88, p=.19)$ decibels, but there was a significant difference in mu-band power (control group $=-0.72( \pm 0.77)$ decibels, TS group $=-0.25( \pm 0.55)$ decibels; $t$ (28) $=-1.95, p=.03$; effect size $=0.7$ ).

\section{Methods: Study 2. Neural antecedents of motor tics}

\section{Participants}

Seventeen individuals (five female) aged between 12 and 26 years (mean age of 18 years) with a confirmed diagnosis of Tourette syndrome took part in the study. Four of these participants had taken part in Study 1. Participants were recruited from Tourette syndrome clinic at the Queen's Medical Centre, Nottingham and also through the UK charity Tourettes Action. All participants (and their parent/guardian where appropriate) provided informed consent to participate in the study which was approved by an appropriate local ethical review committee. Participants with co-occurring conditions were not excluded. One participant's data had to be rejected due to there being no intervals between their tics. The 16 participants that remained included four females aged between 12 and 23 years (mean age of 17.4 years). Of the sixteen subjects, five were currently taking medication. Details from participants can be found in Table 2 . All of the participants were currently exhibiting high levels of motor tics. The mean motor tic score for the group was $18.73 / 25$ ( \pm 3.47 , range $13-23)$.

\section{EEG data collection}

EEG data collection was identical to that described for Study 1. Two EMG electrodes were placed over the orbicularis oris in order to record the onset of tics involving mouth and nose movements. Time windows of -1.2 to $0.5 \mathrm{~s}$, time-locked to the tic onset were extracted. The average number of epochs across participants was 42 .

\section{Experimental procedure}

During EEG recording, all participants' eye and facial movements were video recorded for off-line analysis of eye and facial tics. At the start of the video recording, participants were 


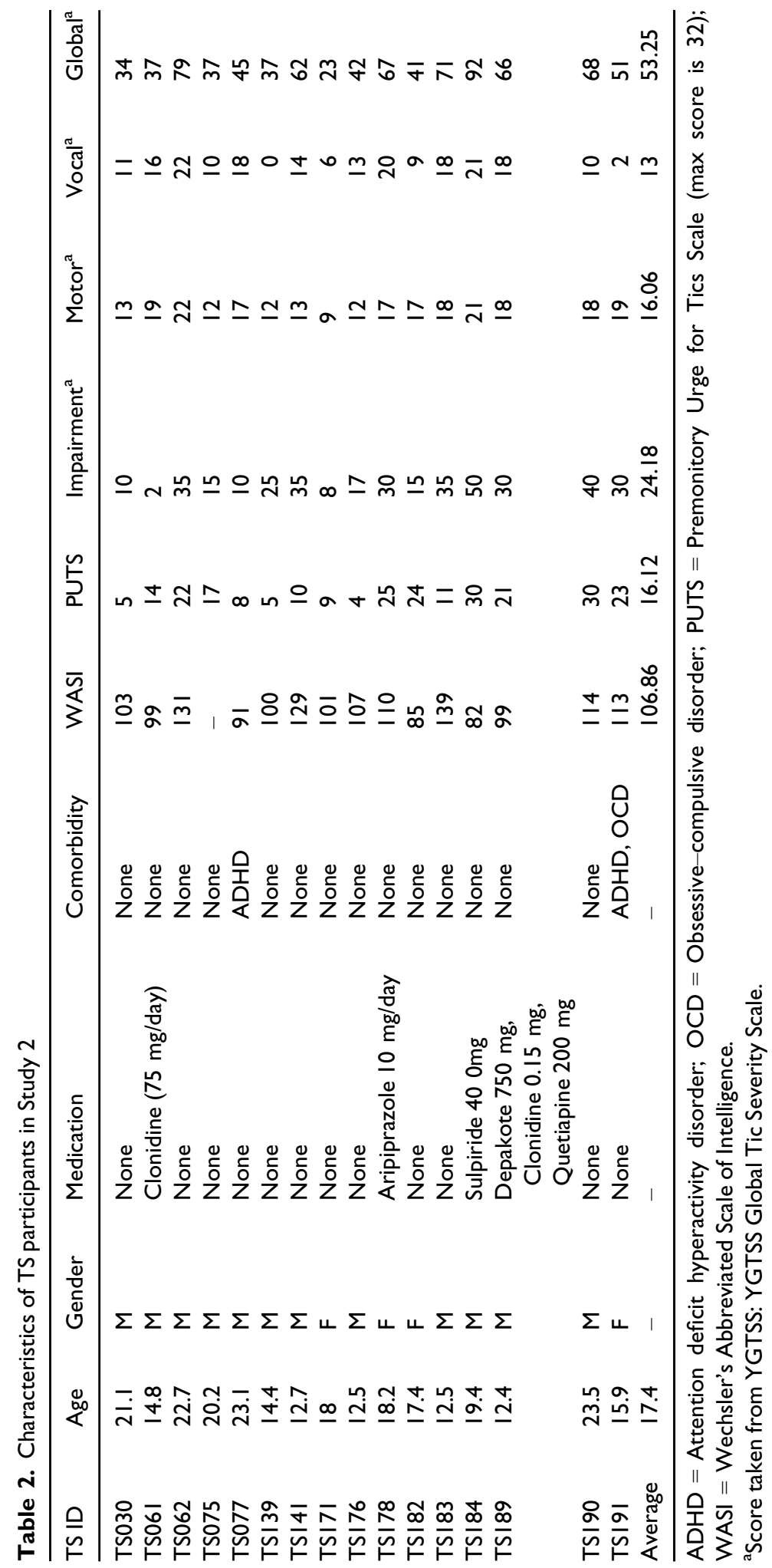


asked to sit in a relaxed posture and were instructed not to suppress their tics. Testing took $\sim 28$ min with three small breaks every $7 \mathrm{~min}$.

\section{Selection and marking of tic events}

EEG data were synchronized with the video recorded during the testing. Off-line video analysis was conducted using QuickTime 7 Pro player allowing frame-by-frame analysis (maximum error range $20 \mathrm{~ms}$ ). Once the EEG recording was synchronized with the video, all tics that occurred in a period at least $1 \mathrm{~s}$ from the last recorded tic, or from a voluntary movement, were marked in the EEG data. This process was performed twice for each participant to ensure reliability of identifying tic onsets. As a result, only tics that were preceded by at least one second of resting state (i.e., no movement observed) and could be clearly identified in the EEG data were marked. Because of these conservative requirements, some tics, particularly those that occurred in a sequence separated by less than a second, may not have been identified as individual events. That said, all of the participants exhibited large number of recordable tics during the video recording. The mean number of recorded tics for the group was $42.2 \pm 20.4$, with a range of $17-84$ tics (Median $=37$, inter-quartile range $=28.5-51.8)$.

\section{EEG data analysis}

As outlined above, analyses of the EEG data were conducted to obtain ERP and ERSP for the electrodes located on the scalp over the sensorimotor cortex (i.e., average ERP or ERSP from electrodes C3, C1, C2, and C4).

\section{Results}

We conducted a time-frequency analyses for mu-band (Figure 5A) and beta-band (Figure 5B) power (ERSP), time-locked to the onset of tics ( $0 \mathrm{~ms})$. These time-frequency analyses demonstrate that, in contrast to voluntary movements, there was no substantive desynchronization of power immediate prior to, or at the time of tic onset, for either mu or beta rhythm (see Figure 3). Figure 5 does appear to show an apparent increase in ERSP across multiple bandwidths after movement onset; however, it is important to recognize these this is unlikely to reflect an event-related increase in power, but instead almost certainly reflects muscle activity (artefact) generated as a consequence of tic-related facial/head movement and should therefore be discounted and will not be discussed further.

\section{Discussion}

We examined the neural antecedents of voluntary movements and of motor tics using EEG-recording techniques. Our results can be summarized as follows. First, we observed that time-frequency analysis of movement-related beta-band oscillation power revealed that for voluntary movements both the TS group and controls exhibited an event-related desynchronization (ERD) that commenced prior to movement onset. However, a similar time-frequency analysis of mu-band oscillation power revealed that, whereas the healthy control group exhibited a clear mu-band ERD that commenced at movement onset, this was not observed for the TS group. Second, time-frequency analyses of both mu-band and 
(a)

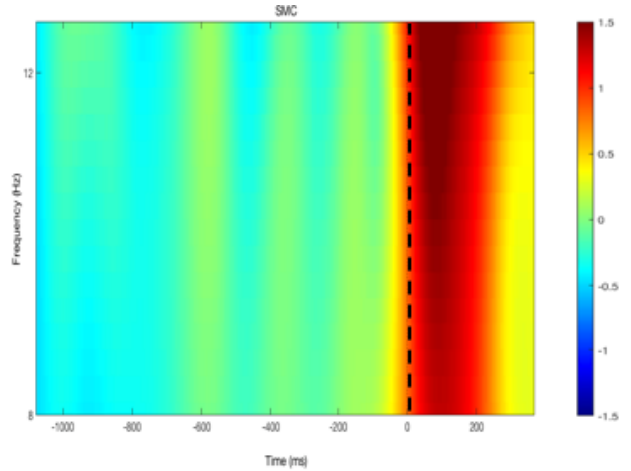

(b)

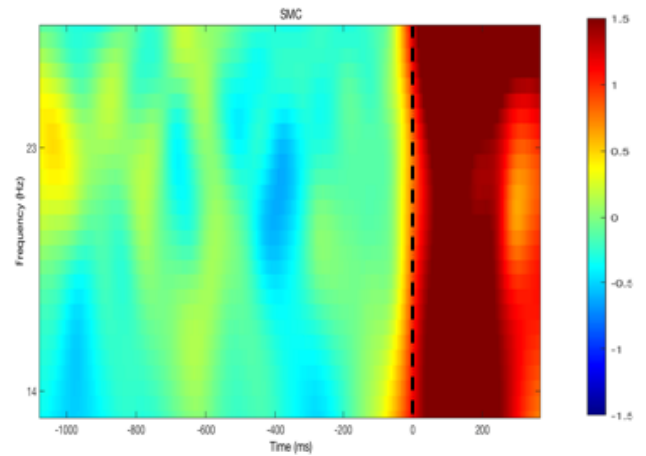

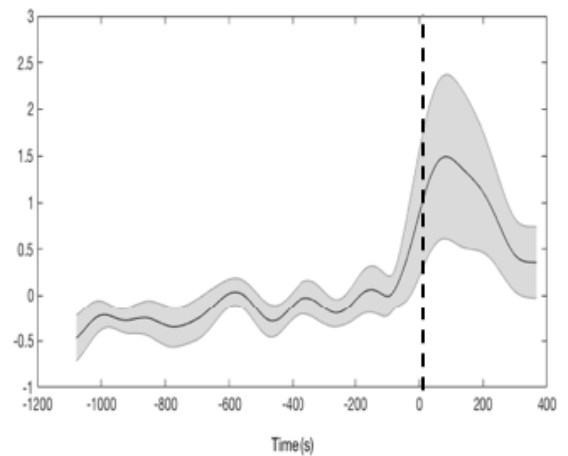

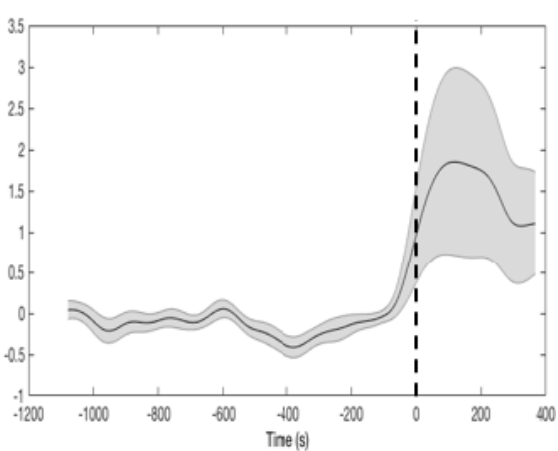

Figure 5. A. Left panel: Time-frequency plots in mu-band $(8-13 \mathrm{~Hz})$ for the average electrodes on the SMC. Right panel: Values of power for the average electrodes on the SMC. B. Left panel: Time-frequency plots in beta-band $(13-30 \mathrm{~Hz})$ for the average electrodes on the SMC. Values of power for the average electrodes on the SMC in mu-band $(8-13 \mathrm{~Hz})$. Right panel: Values of power for the average electrodes on the SMC in beta-band $(13-30 \mathrm{~Hz}) .0$ indicates the onset of the tic.

beta-band power prior to the onset of tic movements confirmed that there was no evidence of any ERD immediately prior to the onset of tics.

\section{Absence of beta- and mu-band oscillations prior to tics in TS}

Neural oscillations of the brain's electromagnetic activity reflect the synchronized firing of populations of neurons, and it is known that GABA-mediated interneurons play a critical role in co-ordinating the synchronized activity of populations of pyramidal neurons that give rise to brain oscillations (Schnitzler \& Gross, 2005). Two frequency bands are particularly relevant to the occurrence of tics in TS: Mu $(8-12 \mathrm{~Hz})$ and beta $(13-30 \mathrm{~Hz})$, which have long been associated with sensorimotor function (Armstrong, Sale, \& Cunnington, 2018); become de-synchronized when a movement is initiated (Schnitzler \& Gross, 2005); and, have been linked to maintaining the current motor set (Engel \& Fries, 2010). However, a more recent proposal is that beta-band oscillation power reflects an internal estimate of the likelihood of the need for a novel voluntary action, and effectively signals the currently level of motor readiness (Jenkinson \& Brown, 2011). Furthermore, it is argued that beta-band oscillations are linked to net dopamine levels within key sites in 
cortical-striatal-thalamic-cortical motor networks (Jenkinson \& Brown, 2011). Within this context, it is of interest to note that Parkinson's disease - a disorder that is associated with a poverty of spontaneous movements (Akinesia), slowed movements (Bradykinesia), and is caused by a loss of dopamine cells within the substantia nigra - is associated with abnormally increased power in beta-band oscillations, that is reduced by dopamine medication and deep-brain stimulation. Moreover, the extent to which bradykinesia improves following drug treatment is related to the extent to which beta-band oscillation power is reduced within the cortical-striatal-thalamic-cortical motor circuit (Jenkinson \& Brown, 2011). By contrast, Tourette syndrome - a disorder that is associated with the occurrence of involuntary movements and vocalizations (tics), and is associated with increased dopamine activity within the cortical-striatal-thalamic-cortical motor circuit (Buse et al., 2013) - is shown in the current study to be associated with an absence of betaand mu-band desynchronization ahead of the occurrence of motor tics. While this finding is consistent with the results of previous EEG studies that demonstrate an absence of movement-related ERP prior to tics in TS, it is nonetheless rather puzzling as to why no movement-related EEG signals (i.e., ERD) are observed immediately prior to, or during, tic onset, as functional magnetic resonance imaging (fMRI) studies of this issue have clearly demonstrated increased brain activity in sensorimotor cortex both immediately before tic onset and at tic onset. Specifically, an early study examined brain activity occurring $2 \mathrm{~s}$ before tic onset and also at tic onset, and found widespread increases in activation in paralimbic areas such as anterior cingulate cortex and insular, the supplementary motor area and parietal operculum $2 \mathrm{~s}$ prior to tic, whereas during tic execution activity was observed in primarily sensorimotor cortex and the cerebellum (Bohlhalter et al., 2006). Similarly, a more recent study with a far larger sample size largely confirmed this finding and reported increased activity within the sensorimotor cortex both $1 \mathrm{~s}$ before, and at, tic onset (Neuner et al., 2014). These studies confirm that tic generation is associated with a set of brain areas that include, in particular, the insula and cingulate cortices, the cerebellum, and basal ganglia nuclei, which may indicate that the generation of tics in TS may involve brain activity that is not typically observed for volitional movements. This is supported by the striatal disinhibition animal model of TS. In this model, tic-like movements are produced following localized injections of a GABA-antagonist into the striatum of the animal (Bronfeld et al., 2013; McCairn, Iriki, \& Isoda, 2013; Worbe et al., 2009; Worbe et al., 2013). McCairn and colleagues conducted multisite, multielectrode electrophysiological recordings of single-unit activity and local field potentials (LFPs) from a number of brain areas in non-human primates, including the cerebellum; basal ganglia; and primary motor cortex, while the animal exhibited tic-like movements. A key finding from their study was that, while changes in LFPs preceded the occurrence of tic-like movements in many of the brain areas recorded from with different latencies, the occurrence of tic-like movements was most closely associated with LFPs in the primary motor cortex and cerebellum (McCairn et al., 2013). The authors propose that while striatal disinhibition might be a trigger for tic-like movements, the primary motor cortex, and in particular the cerebellum, may act as a gate to release overt tic-like movements (McCairn et al., 2013).

One difference between volitional movements and tics is that tics are most often preceded by a premonitory sensory/urge phenomena (PU) which are consciously experienced as a strong urge for motor discharge and have been thought of as a driver for tics. Importantly, in the current study, all of the individuals with TS reported experiencing PU. PU have been particularly linked to the insular cortex, and with the mid-cingulate cortical region that has been associated with the triggering of actions in response to PU 
(Jackson et al., 2011). In this context, it is important to note that brain activity within the mid-cingulate cortex increases immediately prior to the execution of tics (Bohlhalter et al., 2006; Neuner et al., 2014) and that there are alterations in grey matter (GM) volume throughout mid and anterior cingulate cortex that are correlated with clinical measures of tic severity (O'Neill et al., 2019). Furthermore, direct electrical stimulation of the midcingulate cortex is sufficient to induce movements, including goal-directed actions, with no evidence that electrical stimulation of this region induces a phenomenological experience of an 'urge-to-move' (Caruana et al., 2018; Trevisi et al., 2018). Finally, recent brain imaging studies investigating differences in seed-based structural covariance networks (SCNs) in individuals with TS compared to matched controls have demonstrated that the SCNs linked to the insula (Jackson, Loayza, et al., 2020) and mid-cingulate cortex (Jackson, Sigurdsson et al., 2020), and the motor cerebellum (Sigurdsson, Jackson, Jolley, Mitchell, \& Jackson, 2020) have substantially different patterns of structural covariance compared to typically developing individuals. Importantly, each study indicates that structural covariance is substantially increased between the insula and mid-cingulate cortex in individuals with TS relative to controls. Taken together, these studies lend support to the proposal that the contribution of paralimbic, sub-cortical, and cerebellar regions may play a far greater role in the generation of tics in TS, than they necessarily do in the generation of volitional movements.

\section{Difference between mu- and beta-band oscillations during voluntary movements in TS}

As noted above, in the current study, we observed that the beta-band EEG signals recorded from the scalp over the sensorimotor cortex prior to volitional movements were similar for individuals with TS and typically developing individuals. Specifically, the magnitude of the beta-band ERD observed for the TS group did not differ from that of the matched control group. By contrast, analysis of mu-band EEG power revealed that while the healthy control group exhibited a clear mu-band ERD that commenced at movement onset, this was not observed for the TS group, and mu power for the pre-movement period was significantly different between the groups. Below we discuss two potential explanations for this observation, both of which can be linked to altered physiological inhibition in TS. Before doing so it is, however, important to note that, given that neural oscillations of the brain's electromagnetic activity reflect the synchronized firing of populations of neurons, we should be cautious in attributing particular functions to specific EEG band oscillations.

First, it has been suggested that TS is associated with increased levels of sensorimotor noise that accompany tics and result in it being more difficult for individuals with TS to distinguish tics from volitional movements (Ganos et al., 2015). Evidence in support of this proposal comes from a recent study that examined forward model updating in young adults with TS (Kim, Jackson, Dyke, \& Jackson, 2019). Thus, it may be that sensorimotor processes, including the timing of movement-related brain oscillations, are less precise in individuals with TS; perhaps as a consequence of alterations in GABA-mediated inhibitory processes which are known to play a key role co-ordinating the synchronized activity of populations of pyramidal neurons that give rise to brain oscillations (Prokic, Stanford, Woodhall, Williams, \& Hall, 2019; Schnitzler \& Gross, 2005), and are reported to be abnormal in individuals with TS (Gilbert et al., 2004; Kalanithi et al., 2005; Lerner et al., 2012; Orth \& Rothwell, 2009; Puts et al., 2015). Specifically, there are now multiple lines of evidence to indicate that TS is associated with altered GABA function within corticalstriatal-thalamo-cortical motor networks. Post-mortem investigations of TS have demonstrated substantial decreases $(\sim 50 \%)$ in the number of GABAergic interneurons 
found within the striatum (Kalanithi et al., 2005), and positron emission tomography (PET) imaging has revealed widespread alterations in $\mathrm{GABA}_{\mathrm{A}}$ receptor binding in $\mathrm{TS}$, in particular within the striatum, thalamus, insula, and primary somatosensory cortex (Lerner et al., 2012). In addition, transcranial magnetic stimulation (TMS) studies of physiological inhibition within the primary motor cortex have demonstrated reduced $\mathrm{GABA}_{\mathrm{A}}$ receptor dependent short intracortical inhibition (Gilbert et al., 2004; Orth \& Rothwell, 2009) and reduced interhemispheric inhibition (IHI) in TS (Bäumer et al., 2010), and a magnetic resonance spectroscopy (MRS) study has reported decreased concentrations of GABA within the primary sensorimotor cortex in children with TS (Puts et al., 2015). In the current study, and consistent with the proposal for increased sensorimotor noise in TS, we observed in Study 1 that, while the mean timing of movement-related desynchronization (ERD) did not differ from controls in the TS group, the variability in the timing of the peak ERD relative to movement onset was larger in the TS group for both mu-and beta-band oscillations (i.e., the CoV for the TS group was almost twice that of the CS group). However, it is important to note that this difference was not statistically significant and should be treated with caution. We suggest that it would be interesting to investigate this further in a larger sample.

While both mu- and beta-band oscillations are associated with the initiation of voluntary movements, it is important to recognize that they may nonetheless each index different sensorimotor functions. As noted above, beta-band oscillations are thought to play a role in movement preparation by reflecting an internal estimate of the likelihood of the need for a novel voluntary action and signalling the current state of motor readiness (Jenkinson \& Brown, 2011). As such, it is likely that beta-band desynchronization is associated with the disinhibition (activation) of movement-related neuronal populations. By contrast, mu-band oscillations are thought to play a key role in inhibiting neuronal populations that might otherwise interfere with movement selection (Brinkman et al., 2016; Brinkman, Stolk, Dijkerman, de Lange, \& Toni, 2014; Jensen \& Mazaheri, 2010).

Previous studies have reported that individuals with TS exhibit reduced cortical excitability in the motor cortex during movement preparation compared to healthy controls (Draper et al., 2013; Heise et al., 2010; Jackson et al., 2013), and fMRI studies have also shown a decreased BOLD signal in the motor cortex during execution of movement in individuals with TS compared to healthy controls (Jackson et al., 2011; Roessner et al., 2012; Thomalla et al., 2014). In the current study, we found that when executing voluntary movements, the mean amplitude of movement-related beta-band ERDs, while somewhat reduced in the TS group, did not differ significantly to that of the matched health control group. By contrast, we observed that while the control group exhibited a significant movement-related mu-band ERD, this was not observed for the TS group, and the magnitude of the ERD was significantly different across the groups. These findings suggest that while processes involved in the disinhibition of movement-related populations within the sensorimotor cortex may be working effectively in individuals with TS, those neural mechanisms involved in suppressing processes that might otherwise compete with movement generation are working less efficiently in TS. This finding is entirely consistent with the pathophysiology of TS which has been linked with reduced physiological inhibition within the cortico-striatal-thalamo-cortical motor circuit (Albin \& Mink, 2006). Specifically, it is consistent with the finding that short-interval intracortical inhibition (SICI), a phenomenon that is thought to be dependent upon GABA interneurons, and mediated by $\mathrm{GABA}_{\mathrm{A}}$ receptors, is significantly reduced in individuals with TS (Gilbert et al., 2004; Orth \& Rothwell, 2009). It is also consistent with the finding that somatosensory function (i.e., tactile detection and adaptation tasks) was impaired, 
and GABA concentrations within primary sensorimotor cortex reduced, in children with TS compared to a matched control group (Puts et al., 2015). Furthermore, this study showed that sensorimotor GABA concentrations were associated with both the severity of motor tics and altered somatosensory function in the children with TS. Together, these studies indicate that individuals with TS may exhibit an impairment in physiological inhibition mechanisms that would likely impact on their ability to effectively inhibit competing neuronal populations during movement preparation.

In summary, the current study has confirmed that movement-related mu- and betaband oscillations, that have been linked to the initiation of volitional movements, are not observed prior to tics in individuals with TS. We interpret this effect as reflecting the greater involvement of a network of brain areas, including the insular and cingulate cortices, basal ganglia nuclei, and the cerebellum, in the generation of tics in TS. By contrast, beta-band desynchronization is demonstrated to occur when individuals with TS initiate voluntary movements, but, in contrast to healthy controls, desynchronization of mu-band oscillations is not observed during the execution of voluntary movements for individuals with TS. We interpret this finding as reflecting a dysfunction of physiological inhibition in TS, thereby contributing to an impaired ability to suppress neuronal populations that may compete with movement preparation processes.

\section{Study limitations}

One factor that should be considered when evaluating our findings is that the movements in Studies 1 and 2 were not identical. Specifically, in Study 1, which examined the EEG antecedents of voluntary movements, all participants executed a movement using their index finger. By contrast, in Study 2, which investigated the EEG antecedents of tics in individuals with TS, the movements were a heterogeneous mixture of predominately facial movements. It is important to note that these studies were not intended to be directly comparable studies as neurotypical individuals do not exhibit tics. By contrast, Study 1 was intended to confirm, as had been previously reported, that the individuals in our study did indeed exhibit desynchronization of movement-related brain oscillations ahead of a voluntary movement, whereas Study 2 was intended to investigate if similar desynchronization of movement-related brain oscillations occurs ahead of tics in TS. In the latter case, we were able to confirm the previous finding of Obeso et al. (1981) that the EEG signature for voluntary movements was not observed prior to the occurrence of tics in TS.

A second factor to consider is that the number of participants in this study, while larger than previously reported studies, is relatively small. This reflects the difficulty of recruiting large numbers of individuals with TS to neuroimaging studies, which are often perceived as difficult for them (due to their being rejected from participating due to their tics). A related factor is that while our sample of TS participants presented for the most part without significant commonly co-occurring conditions (such as OCD or ADHD), those taking medication were taking a variety of medications. For this reason, we can say very little about the effect of comorbidity or medication.

Third, our study was conducted using a sample of individuals with TS aged between 12 and 26 years (mean age of 18 years). As this is a period of considerable developmental change, it would have been useful to conduct a developmental analyses of the effects reported in paper. However, with a small sample, this is not possible and we note this as a limitation. 


\section{Acknowledgements}

This work was supported by Tourettes Action (UK) and the NIHR Nottingham Biomedical Research Centre. The views expressed are those of the authors and not necessarily those of the NHS, the NIHR or the Department of Health. We thank Tourettes Action (UK) for assisting with participant recruitment.

\section{Conflicts of interest}

All authors declare no conflict of interest.

\section{Data Availability Statement}

The data that support the findings of this study are available on request from the corresponding author [SRJ]. The data are not publicly available due to ethical restrictions and their containing information that could compromise the privacy of research participants.

\section{References}

Albin, R. L., \& Mink, J. W. (2006). Recent advances in Tourette syndrome research. Trends in Neurosciences, 29, 175-182. https://doi.org/10.1016/j.tins.2006.01.001

Armstrong, S., Sale, M. V., \& Cunnington, R. (2018). Neural oscillations and the initiation of voluntary movement. Frontiers in Psychology, 9, 2509. https://doi.org/10.3389/fpsyg.2018.02509

Bäumer, T., Thomalla, G., Kroeger, J., Jonas, M., Gerloff, C., Hummel, F. C., . . Münchau, A. (2010). ... Münchau A. Interhemispheric motor networks are abnormal in patients with Gilles de la Tourette syndrome. Movement Disorders, 25, 2828-2837. https://doi.org/10.1002/mds. 23418

Benjamini, Y., \& Hochberg, Y. (1995). Controlling the false discovery rate - a practical and powerful approach to multiple testing. Journal of the Royal Statistical Society Series B-Statistical Methodology, 57(1), 289-300. https://doi.org/10.1111/j.2517-6161.1995.tb02031.

Bohlhalter, S., Goldfine, A., Matteson, S., Garraux, G., Hanakawa, T., Kansaku, K., ... Hallett, M. (2006). Neural correlates of tic generation in Tourette syndrome: An event-related functional MRI study. Brain, 129, 2029-2037. https://doi.org/10.1093/brain/awl050

Brinkman, L., Stolk, A., Dijkerman, H. C., de Lange, F. P., \& Toni, I. (2014). Distinct roles for alpha-and beta-band oscillations during mental simulation of goal-directed actions. Journal of Neuroscience, 34, 14783-14792. https://doi.org/10.1523/JNEUROSCI.2039-14.2014

Brinkman, L., Stolk, A., Marshall, T. R., Esterer, S., Sharp, P., Dijkerman, H. C., . . Toni, I. (2016). Independent causal contributions of alpha-and beta-band oscillations during movement selection. Journal of Neuroscience, 36, 8726-8733. https://doi.org/10.1523/JNEUROSCI. 0868-16.2016

Bronfeld, M., Israelashvili, M., \& Bar-Gad, I. (2013). Pharmacological animal models of tourette syndrome. Neuroscience and Biobehavioral Reviews, 37(6), 1101-1119. https://doi.org/10. 1016/j.neubiorev.2012.09.010.

Buse, J., Schoenefeld, K., Münchau, A., \& Roessner, V. (2013). Neuromodulation in Tourette syndrome: Dopamine and beyond. Neuroscience \& Biobehavioral Reviews, 37, 1069-1084. https://doi.org/10.1016/j.neubiorev.2012.10.004

Caruana, F., Gerbella, M., Avanzini, P., Gozzo, F., Pelliccia, V., Mai, R., . . Rizzolatti, G. (2018). Motor and emotional behaviours elicited by electrical stimulation of the human cingulate cortex. Brain, 141, 3035-3051. https://doi.org/10.1093/brain/awy219

Cavanna, A. E., Black, K. J., Hallett, M., \& Voon, V. (2017). Neurobiology of the premonitory urge in tourette's syndrome: Pathophysiology and treatment implications. The Journal of 
Neuropsychiatry and Clinical Neurosciences, 29, 95-104. https://doi.org/10.1176/appi.ne uropsych.16070141

Chen, R., Corwell, B., \& Hallett, M. (1999). Modulation of motor cortex excitability by median nerve and digit stimulation. Experimental Brain Research, 129(1), 77. https://doi.org/10.1007/ s002210050938

Chen, R., Yaseen, Z., Cohen, L. G., \& Hallett, M. (1998). Time course of corticospinal excitability in reaction time and self-paced movements. Annals of Neurology, 44(3), 317-325. https://doi.org/ 10.1002/ana.410440306

Cohen, S. C., Leckman, J. F., \& Bloch, M. H. (2013). Clinical assessment of Tourette syndrome and tic disorders. Neuroscience \& Biobehavioral Reviews, 37, 997-1007. https://doi.org/10.1016/j.ne ubiorev.2012.11.013

Draper, A., Jude, L., Jackson, G. M., \& Jackson, S. R. (2013). Motor excitability during movement preparation in Tourette syndrome. Journal of Neuropsychology, 9(1), 33-44. https://doi.org/ $10.1111 /$ jnp. 12033

Duggal, H. S., \& Nizamie, S. H. (2002). Bereitschaftspotential in tic disorders: A preliminary observation. Neurology India, 50, 487-489.

Engel, A. K., \& Fries, P. (2010). Beta-band oscillations-signalling the status quo? Current Opinion in Neurobiology, 2O(2), 156-165. https://doi.org/10.1016/j.conb.2010.02.015

Ganos, C., Asmuss, L., Bongert, J., Brandt, V., Münchau, A., \& Haggard, P. (2015). Volitional action as perceptual detection: Predictors of conscious intention in adolescents with tic disorders. Cortex, 64, 47-54. https://doi.org/10.1016/j.cortex.2014.09.016

Gilbert, D. L., Bansal, A. S., Sethuraman, G., Sallee, F. R., Zhang, J., Lipps, T., \& Wassermann, E. M. (2004). Association of cortical disinhibition with tic, ADHD, and OCD severity in Tourette syndrome. Movement Disorders, 19(4), 416-425. https://doi.org/10.1002/mds.20044

Heise, K.-F., Steven, B., Liuzzi, G., Thomalla, G., Jonas, M., Muller-Vahl, K., . . Hummel, F. C. (2010). Altered modulation of intracortical excitability during movement preparation in Gilles de la Tourette syndrome. Brain, 133, 580-590. https://doi.org/10.1093/brain/awp299

Jackson, S. R., Loayza, J., Crighton, M., Sigurdsson, H. P., Dyke, K., \& Jackson, G. M. (2020). The role of the insula in the generation of motor tics and the experience of the premonitory urge-to-tic in Tourette syndrome. Cortex, 126, 119-133. https://doi.org/10.1016/j.cortex.2019.12.021

Jackson, S. R., Parkinson, A., Jung, J., Ryan, S. E., Morgan, P. S., Hollis, C., \& Jackson, G. M. (2011). Compensatory neural reorganization in Tourette syndrome. Current Biology, 21, 580-585. https://doi.org/10.1016/j.cub.2011.02.047

Jackson, S. R., Parkinson, A., Manfredi, V., Millon, G., Hollis, C., \& Jackson, G. M. (2013). Motor excitability is reduced prior to voluntary movements in children and adolescents with Tourette syndrome. Journal of Neuropsychology, 7(1), 29-44. https://doi.org/10.1111/j.1748-6653. 2012.02033.x

Jackson, S. R., Sigurdsson, H. P., Dyke, K., Condon, M., \& Jackson, G. M. (2020). The role of the cingulate cortex in the generation of motor tics and the experience of premonitory urge-to-tic in Tourette syndrome. bioRxiv.

Jenkinson, N., \& Brown, P. (2011). New insights into the relationship between dopamine, beta oscillations and motor function. Trends in Neurosciences, 34, 611-618. https://doi.org/10. 1016/j.tins.2011.09.003

Jensen, O., \& Mazaheri, A. (2010). Shaping functional architecture by oscillatory alpha activity: Gating by inhibition. Frontiers in Human Neuroscience, 4, 186. https://doi.org/10.3389/ fnhum.2010.00186

Kalanithi, P. S. A., Zheng, W., Kataoka, Y., DiFiglia, M., Grantz, H., Saper, C. B., .. Vaccarino, F. M. (2005). . . Vaccarino FM. Altered parvalbumin-positive neuron distribution in basal ganglia of individuals with Tourette syndrome. Proceedings of the National Academy of Sciences, 102, 13307-13312. https://doi.org/10.1073/pnas.0502624102

Karp, B. I., Porter, S., Toro, C., \& Hallett, M. (1996). Simple motor tics may be preceded by a premotor potential. Journal of Neurology, Neurosurgery and Psychiatry, 61(1), 103-106. https://doi.org/10.1136/jnnp.61.1.103 
Kim, S., Jackson, G. M., Dyke, K., \& Jackson, S. R. (2019). Impaired forward model updating in young adults with Tourette syndrome. Brain, 142(1), 209-219. https://doi.org/10.1093/brain/ awy306

Leckman, J. F., Bloch, M. H., Scahill, L., \& King, R. A. (2006). Tourette syndrome: The self under siege. Journal of Child Neurology, 21(8), 642-649. https://doi.org/10.1177/ 08830738060210081001

Lerner, A., Bagic, A., Simmons, J. M., Mari, Z., Bonne, O., Xu, B., .. Hallett, M. (2012). Widespread abnormality of the $\gamma$-aminobutyric acid-ergic system in Tourette syndrome. Brain, 135, 19261936. https://doi.org/10.1093/brain/aws104

McCairn, K. W., Iriki, A., \& Isoda, M. (2013). Global dysrhythmia of cerebro-basal ganglia-cerebellar networks underlies motor tics following striatal disinhibition. Journal of Neuroscience, 33, 697-708. https://doi.org/10.1523/JNEUROSCI.4018-12.2013

Neuner, I., Werner, C. J., Arrubla, J., StÃ $\mid$ cker, T., Ehlen, C., Wegener, H. P., . . Shah, N. J. (2014). Imaging the where and when of tic generation and resting state networks in adult Tourette patients. Frontiers in Human Neuroscience, 8, 362. https://doi.org/10.3389/fnhum.2014. 00362

Obeso, J. A., Rothwell, J. C., \& Marsden, C. D. (1981). Marsden CD Simple tics in Gilles de la Tourette's syndrome are not prefaced by a normal premovement EEG potential. Journal of Neurology, Neurosurgery and Psychiatry, 44, 735-738. https://doi.org/10.1136/jnnp.44.8. 735

O'Neill, J., Piacentini, J. C., \& Peterson, B. S. (2019). Cingulate role in Tourette syndrome. In Handbook of clinical neurology (Vol. 166, pp. 165-221). Amsterdam, Netherlands: Elsevier.

Orth, M., \& Rothwell, J. C. (2009). Motor cortex excitability and comorbidity in Gilles de la Tourette syndrome. Journal of Neurology, Neurosurgery \& Psychiatry, 80, 29-34. https://doi.org/10. 1136/jnnp.2008.149484

Prokic, E. J., Stanford, I. M., Woodhall, G. L., Williams, A. C., \& Hall, S. D. (2019). Bradykinesia is driven by cumulative beta power during continuous movement and alleviated by gabaergic. Frontiers in Neurology, 10, 1298. https://doi.org/10.3389/fneur.2019.01298

Puts, N. A. J., Harris, A. D., Crocetti, D., Nettles, C., Singer, H. S., Tommerdahl, M., . . Mostofsky, S. H. (2015). Reduced GABAergic inhibition and abnormal sensory symptoms in children with Tourette syndrome. Journal of Neurophysiology, 114, 808-817. https://doi.org/10.1152/jn. 00060.2015

Roessner, V., Wittfoth, M., Schmidt-Samoa, C., Rothenberger, A., Dechent, P., \& Baudewig, J. (2012). Altered motor network recruitment during finger tapping in boys with Tourette syndrome. Human Brain Mapping, 33, 666-675. https://doi.org/10.1002/hbm.21240

Schnitzler, A., \& Gross, J. (2005). Normal and pathological oscillatory communication in the brain. Nature Reviews Neuroscience, 6(4), 285-296. https://doi.org/10.1038/nrn1650

Sigurdsson, H. P., Jackson, S. R., Jolley, L., Mitchell, E., \& Jackson, G. M. (2020). Alterations in cerebellar grey matter structure and covariance networks in young people with Tourette syndrome. Cortex, 126, 1-15. https://doi.org/10.1016/j.cortex.2019.12.022

Thomalla, G., Jonas, M., Bäumer, T., Siebner, H. R., Biermann-Ruben, K., Ganos, C., . . Münchau, A. (2014). ... Münchau A. Costs of control: Decreased motor cortex engagement during a Go/ NoGo task in Tourette's syndrome. Brain, 137(1), 122-136. https://doi.org/10.1093/brain/ awt288

Trevisi, G., Eickhoff, S. B., Chowdhury, F., Jha, A., Rodionov, R., Nowell, M., ... Diehl, B. (2018). Probabilistic electrical stimulation mapping of human medial frontal cortex. Cortex, 109, 336346. https://doi.org/10.1016/j.cortex.2018.06.015

van der Salm, S. M., Tijssen, M. A., Koelman, J. H., \& van Rootselaar, A. F. (2012). The bereitschaftspotential in jerky movement disorders. Journal of Neurology, Neurosurgery and Psychiatry, 83, 1162-1167. https://doi.org/10.1136/jnnp-2012-303081

Worbe, Y., Baup, N., Grabli, D., Chaigneau, M., Mounayar, S., McCairn, K., . . Tremblay, L. (2009). Tremblay L Behavioral and movement disorders induced by local inhibitory dysfunction in primate striatum. Cerebral Cortex, 19, 1844-1856. https://doi.org/10.1093/cercor/bhn214 
Worbe, Y., Sgambato-Faure, V., Epinat, J., Chaigneau, M., Tandé, D., François, C., . . Tremblay, L. (2013). Towards a primate model of Gilles de la Tourette syndrome: Anatomo-behavioural correlation of disorders induced by striatal dysfunction. Cortex, 49, 1126-1140. https://doi.org/ 10.1016/j.cortex.2012.08.020.

Zapparoli, L., Macerollo, A., Joyce, E. M., Martino, D., \& Kilner, J. M. (2019). Voluntary tic suppression and the normalization of motor cortical beta power in Gilles de la Tourette syndrome: An EEG study. European Journal of Neuroscience, 50, 3944-3957.

Received 30 June 2020; revised version received 5 March 2021 